\title{
Book presentation : Mohammed Jadir (ed.), (Préface: J. Lahlan Mackenzie) Fonctionnalisme et description linguistique. Editions Universitaires Européennes, Sarrebruck, Allemagne, 2011, 280p. ISBN 978-613-1-56187-0
}

La prise en compte du paramètre de la langue en tant qu'instrument d'interaction sociale dans l'explication des propriétés de la langue naturelle permet de classifier les théories linguistiques modernes en deux catégories: les théories fonctionnelles et les théories formelles.

Dans les théories fonctionnelles, on adopte l'hypothèse selon laquelle la description de la structure des langues naturelles ne peut s'effectuer que si ladite structure est reliée à la fonction de communication. Dans les théories formelles, la langue est considérée comme un système abstrait dont la fonction principale est 'l'expression de la pensée'; aussi les propriétés linguistiques de ce système peuvent-elles y être traitées indépendamment de sa fonction communicative.

Dans les courants formalistes, la 'compétence' du locuteur-auditeur est conçue comme la connaissance des règles (morpho-syntaxiques, sémantiques et phonétiques) de sa langue (Chomsky 1965). Pour les fonctionnalistes, la 'compétence' est une "compétence communicative » (Hymes 1972), en ce sens que la capacité linguistique du sujet parlant comprend non seulement la possibilité de construire et d'interpréter des expressions linguistiques, mais aussi la possibilité d'employer ces expressions conformément aux conventions de l'interaction verbale prévalant dans une communauté linguistique.

Certains linguistes (e.g. Moutaouakil (1989)) remettent en question la dichotomie 'théories fonctionnelles vs. théories formelles' et proposent de lui substituer la dichotomie 'théories fonctionnelles vs. théories non fonctionnelles'. Pour eux, toutes les théories sont formelles du moment qu'elles mettent en œuvre les mêmes mécanismes logico-mathématiques dans la description, l'explication et la formalisation des propriétés des langues naturelles. Ces propriétés sont prises en charge, dans les théories non fonctionnelles, à travers l'adoption de l'hypothèse de 'l'autonomie de la syntaxe' par rapport à la sémantique et à la pragmatique ('systèmes de croyances' en termes de Chomsky 1977) qui sont conçues comme de simples composantes interprétatives. Dans les théories fonctionnelles, le traitement de la langue s'effectue, dans le cadre d'une interaction entre les différents composants de l'organisation linguistique, de la manière suivante : la pragmatique est un cadre théorique englobant, au sein duquel la sémantique et la syntaxe doivent être étudiées.

Compte tenu du fait que les fonctionnalistes se réclament du principe majeur selon lequel « la fonction détermine la forme » et que les non fonctionnalistes adoptent l'hypothèse de la primauté de la syntaxe sur les autres niveaux d'analyse, il est possible de typifier leurs théories respectives comme suit. Relèvent des théories fonctionnelles la théorie de la 'Perspective Fonctionnelle de la Phrase' de l'Ecole de Prague (e.g. Daneš, Ferbas parmi d'autres) - qui prévoit trois niveaux d'analyse : le niveau de la structure sémantique, le niveau de la structure grammaticale et le niveau de la structure fonctionnelle (thème/rhème) -, la théorie de la Grammaire Fonctionnelle Systémique (GFS) de Halliday - qui comprend trois systèmes linguistiques : le système séman-

\footnotetext{
* Mohammed Jadir

Directeur du Laboratoire Langues

Littératures et Traduction (LALITRA)

Université Hassan II

Mohammedia-Casablanca

Maroc

Mohammedjadir@yahoo.fr
} 
tique de 'transitivité', le système de 'mode' reflétant la fonction interpersonnelle et le système de 'thème' (le thème, le donné, le nouveau) -, la théorie de la Grammaire Fonctionnelle (GF) de Dik - qui prévoit trois niveaux fonctionnels : le niveau interpersonnel/ pragmatique (fonctions pragmatiques (e.g. Topique, Focus), force illocutionnaire...), le niveau représentationnel/sémantique et le niveau structurel -, et enfin la Grammaire du Rôle et de la Référence (GRR) de Van Valin \& Foley qui subsume trois structures : une structure pragmatique ('référentielle'), une structure sémantique (des 'rôles') et une structure syntaxique.

Pour Moutaouakil, même la Sémantique Générative et la Syntaxe Fonctionnelle de Kuno relèvent des théories fonctionnelles. La théorie de la Grammaire Générative et Transformationnelle (GGT) est une illustration de théorie non fonctionnelle où le composant sémantico-pragmatique fournit une interprétation aux structures générées syntaxiquement.

Si le fonctionnalisme, ainsi défini, semble une 'étiquette unificatrice', comment concevoir le fonctionnalisme martinetien qui reconnaît la fonction instrumentaliste de la langue sans pour autant prévoir un 'plan' pragmatique?

Feuillard a consacré son étude à la mise en évidence de caractéristiques fondamentales du fonctionnalisme à partir de 'regards croisés' sur deux courants fondateurs, la Grammaire Fonctionnelle de Dik et la linguistique fonctionnelle de Martinet. Son travail porte successivement sur les principes théoriques, la structure de la « grammaire » et la démarche méthodologique. Au-delà d'un certain nombre de différences, relatives en particulier à la formalisation de la théorie et à la conception sous-jacente de la grammaire, de fortes analogies apparaissent, e.g. la focalisation sur la fonction de communication, l'interaction verbale et sociale, et le respect des données observées.

Le problème débattu dans la contribution de Moutaouakil concerne l'opérationnalisation, en matière de fonctionnalisme, des théories fonctionnelles. L'auteur s'interroge sur la possibilité de passer de 'la théorisation' à 'l'opérationnalisation' des grammaires des théories fonctionnelles au point d'être utilisées dans un bon nombre de secteurs socio-économiques sollicitant le langage (e.g. la traduction, les pathologies langagières, la didactiques des langues, etc.) et d'être mises en rapport avec les grammaires d'autres systèmes non linguistiques (picturaux, mimiques, musicaux, etc.). Moutaouakil prospecte les perspectives qu'offrent les modèles récents de la GF pour la recherche dans ces domaines au Maroc.

Mackenzie a procédé à l'application de certaines notions-clés de la GF au gaélique écossais : les parties du discours, les propriétés spatio-temporelles et aspectuelles, et les fonctions sémantiques, syntaxiques et pragmatiques. L'examen de l'ordre des constituants (langue à ordre postérieur ou antérieur ?) aboutira à une harmonie syntaxique de la langue. Le problème de la distinction entre formes 'dépendantes' et 'indépendantes' trouve son explication dans le placement ou nonplacement du verbe dans la position P1.

Vet et Kampers-Manhe défendent l'idée de la mise en parallèle des emplois du Futur Simple (FS) avec ceux du Futur du Passé (FP), étiquette plus propice pour le conditionnel. Ils considèrent la valeur temporelle de ces formes comme le sens primaire. Ainsi conçoivent-ils trois types d'emplois pour les deux temps : un emploi temporel, un emploi modal et un emploi illocutionnaire. Dans son étude, El Hamraoui argumente en faveur du fait que le prédicat verbal en arabe marocain peut être représenté dans un cadre prédicatif sous forme d'une 'mesure', conçue comme un radical résultant de la lexicalisation du schème et de la racine qui sont attestés en arabe moderne.

Dans leur communication, Hannay et Kroon cherchent à éclairer la notion d'acte en le définissant comme une unité stratégique et non comme une unité conceptuelle tout en en présentant une taxinomie des actes stratégiques. Ils arguent de la corrélation, quoique non bijective, entre les actes discursifs et les unités intonationnelles et ponctuationnelles. Les auteurs considèrent l'implication de leur analyse pour une formalisation dans le cadre de la GF de Discours (GFD). L'objectif de Jadir consiste à présenter et à évaluer les contributions les plus représentatives des fonctionnalistes qui ont abouti à 'l'approche expansionniste' et à 'l'approche modulaire', ainsi 
qu'à argumenter en faveur de l'hypothèse du 'parallélisme croissant' qui se veut un compromis entre les deux approches précédentes.

L'article de Cornish a pour objectif de montrer que les propriétés morpho-syntaxiques, lexicales, sémantiques, référentielles et prosodiques des propositions thétiques, découlent de l'absence de prédication et son corollaire, l'absence de topique, qui les caractérise. Cornish considère que le modèle de la GFD (Hengeveld \& Mackenzie 2006, 2008) peut fournir une meilleure description et explication des énoncés thétiques en vertu de l'organisation des niveaux (e.g Interpersonnel, Représentationnel...) prévus dans la dérivation d'un énoncé.

Kembouche se propose dans son article de déterminer le fonctionnement morphologique et syntaxique des formants vocaliques /a $i \mathrm{u} /$ en arabe. La démarche fonctionnaliste adoptée - au sens de Martinet - permet de mettre en évidence la dynamique de la langue arabe et partant d'établir des fonctionnements différents selon l'idiome considéré, classique ou moderne. Adoptant le principe fonctionnel de la pertinence communicative et la commutation, F. Bentolila tente de joindre la rigueur scientifique à la simplification dans le traitement des faits linguistiques à des fins didactiques. Aussi a-t-il abordé les thèmes d'unités et de classes après avoir évoqué certains problèmes relatifs à la schématisation et à la terminologie.

Il va sans dire que ce volume comporte deux parties. La première est réservée aux contributions s'inscrivant dans le cadre de la GF de Dik (1989-1997) ou dans sa version récente remaniée, la GFD (Hengeveld 2004 et Hengeveld \& Mackenzie 2006-2008). La seconde est consacrée aux travaux d'obédience martinetienne (e.g. Martinet 1960, 1985-1989; Martinet ed. 1979). L'évaluation interne de certains modèles théoriques fonctionnels, le test de leur degré d'adéquation typologique, descriptive et explicative à travers leur application à des phénomènes linguistiques, etc. semblent être les caractéristiques communes à la majorité des articles.

L'objectif de ce collectif, comme du laboratoire 'Théories fonctionnelles des Langues (T $f \mathrm{~L})$ ' de la Faculté des Lettres et des Sciences Humaines de Mohammedia qui a organisé en juin 2008 un colloque international sous le thème fonctionnalisme et description linguistique, est d'établir un dialogue fructueux entre les deux courants fonctionnalistes ; dialogue déjà entamé par Feuillard qui, dans son étude comparative, dégage plus d'affinités qu'elle n'en tire de dissemblances.

Nos vifs remerciements vont à Mme la Doyenne de la Faculté des Lettres et des Sciences Humaines de Mohammedia pour le soutien qu'elle a bien voulu apporter à notre activité scientifique, aux collègues du Département de Langue et de Littérature Françaises qui y ont participé ainsi qu'aux chercheurs qui nous ont fourni les contributions sollicitées pour enrichir le présent volume. Il s'agit de Co Vet \& Brigitte Kampers-Manhe (Université de Groningue, Pays-Bas), Ahmed Moutaouakil (Université Mohammed V), Francis Cornish (CNRS \& Université de Toulouse II), Mike Hannay \& Caroline Kroon (Université Libre d'Amsterdam), John-Lachlan Mackenzie (Université Libre d'Amsterdam \& Institut ILTEC, Lisbonne) et Colette Feuillard \& Fernand Bentolila (Université René Descartes-Sorbonne).

Nous sommes également redevable à Francis Cornish et John-Lachlan Mackenzie pour les soins qu'ils ont bien voulu apporter à ce volume, à Monique Nicolas (ISIT et Paris XIII) pour avoir accepté de lire une première version de ce collectif et enfin à Kheira Kembouche, Mohamed Bouideh et Ahmed El Hamraoui, membres du Laboratoire LALITRA (équipe de recherche Théories fonctionnelles des Langues $(\mathrm{T} f \mathrm{~L})$ ), pour leur contribution à l'organisation du colloque et à la réalisation de ce travail.

\section{Bibliographie}

Chomsky, N. 1965: Aspects de la théorie syntaxique, Paris : Seuil.

Chomsky, N. 1977: Questions de sémantique, Paris : Seuil.

Dik, Simon C. 1979 (1 ère éd. 1978): Functional Grammar. London : Academic Press.

Dik, Simon C. 1989: The theory of Functional Grammar. Part, 1. The structure of the clause. Dodrecht : Foris. 
Jadir, M. 2005: La cohérence du discours en Grammaire Fonctionnelle. Le cas du texte narratif (Préface de Machtelt Bolkestein). Rabat : Editions Bouregreg.

Jadir, M. 2006: Grammaire Fonctionnelle et paramètres textuels. (Préface de Co Vet). Rabat : Editions Bouregreg.

Hengeveld, K. 2004a: The architecture of a Functional Discourse Grammar. In J.L. Mackenzie \& M. A. Gómez-González (éds.), A New Architecture for Functional Grammar. Berlin: Mouton de Gruyter, 1-21.

Hengeveld, K./Mackenzie, J.L. 2006: Functional Discourse Grammar. In K. Brown (éd.), Encyclopedia of Language and Linguistics (2 $2^{\text {nde }}$ édition) Vol. IV. Oxford : Elsevier, 668-676.

Hengeveld, K/Mackenzie, J.L. 2008: Functional Discourse Grammar. Oxford : Oxford University Press.

Hymes, O. 1972: On communicative competence. In Pride, J. B. \& J. Holmes (eds.). Sociolinguistics. Hardmonds : Penguin, 269-293.

Moutaouakil, A: Introduction à la linguistique fonctionnelle (en arabe), Rabat : Okad.

Mackenzie, J.L. 2003: L'utilisation de la grammaire fonctionnelle pour l'analyse de la langue parlée. In Jadir Mohammed (éd.), Développements récents en grammaire fonctionnelle. Mohammedia : Université Hassan II-Mohammedia, 13-28.

Martinet, A. 1956: La description phonologique avec application au parler franco-provençal d'Hauteville. Genève : Droz. Paris : Société de publications romanes et françaises.

Martinet, A. 1960: Eléments de linguistique générale. Paris : Armand Colin.

Martinet, André (sous la dir. de): 1979. Grammaire fonctionnelle du français. Paris : Didier.

Martinet, A. 1985: Syntaxe générale. Paris : Armand Colin.

Martinet, A. 1989: Fonction et dynamique des langues. Paris : Armand Colin. 